Annuaire suisse de politique de développement

13 | 1994

Annuaire Suisse - Tiers Monde 1994

\title{
VI. Coopération au développement
}

\section{(2) OpenEdition}

1 Journals

Édition électronique

URL : http://journals.openedition.org/aspd/1036

DOI : 10.4000/aspd.1036

ISSN : 1663-9669

Éditeur

Institut de hautes études internationales et du développement

\section{Édition imprimée}

Date de publication : 1 janvier 1994

Pagination : 135-154

ISSN : 1660-5934

\section{Référence électronique}

« VI. Coopération au développement », Annuaire suisse de politique de développement [En ligne], 13

1994, mis en ligne le 19 décembre 2012, consulté le 08 septembre 2020. URL : http://

journals.openedition.org/aspd/1036 ; DOI : https://doi.org/10.4000/aspd.1036

(c) The Graduate Institute I Geneva 


\section{Coopération au développement}

L'adhésion de la Suisse à la Banque mondiale et au Fonds monétaire international et la Conférence de Rio sur l'environnement ont marqué en 1992 la coopération suisse au développement. Ces événements engendrent en effet des répercussions durables sur la coopération suisse: l'adhésion à la Banque mondiale et au FMI implique un engagement multilatéral supplémentaire, tant du point de vue financier que pour ce qui est du personnel; la mise en oeuvre de l'Agenda 21, adopté par la Conférence de Rio sur l'environnement, nécessite une réorientation de nombre de projets et de programmes de la coopération suisse au développement et un changement de mentalité de la part des responsables. Pour que les projets prennent plus en compte l'aspect durable et le respect de l'environnement, on devra améliorer lacollaboration entre les divers acteurs suisses-les divers services de l'administration fédérale et les organismes privés - ainsi qu'avec les partenaires dans les pays en développement. Le mot d'ordre est icid'accroître la cohérence des politiques suisses à l'égard du Sud (commerce extérieur, flux de capitaux, exportations d'armes, agriculture, migration, coopération scientifique). Pour poursuivre l'objectif de développement durable - dans les domaines économique, écologique, politique et institutionnel - la coopération publique au développement devra appliquer de nouveaux modes de travail et de nouveaux instruments, viser un travail interdisciplinaire, une collaboration plus étroite avec les oeuvres d'entraide privées et avec les partenaires privés dans les pays en développement et appuyer le processus de démocratisation. La Confédération soutient l'ajustement structurel dans les pays en développement en finançant des réformes économiques, surtout dans le secteur financier, mais aussi en favorisant l'"ajustement social". Les années 1992 et 1993 ont été marquées par une recrudescence des guerres et des conflits et par l'aggravation des violations des droits de l'homme. L'aide humanitaire de la Suisse s'étend au monde entier; au cours de la période considérée, elle a notamment été déployée en Somalie, au Sud de l'Afrique et en ex-Yougoslavie. En 1993, le Comité d'aide au développement de l'OCDE a examiné la coopération suisse au développement. Comme les années précédentes, le comité a jugé qu'elle est d'excellente qualité, mais que son envergure ne reflète pas la capacité économique de la Suisse. (1) 


\section{Aide publique au développement}

Les crédits-cadres (crédits de programme ou d'engagement), que le Parlement accorde en général pour quatre ans, constituent le cadre financier de l'aide publique au développement (APD). Ces crédits se répartissent selon le type d'aide accordée (voir tableau 15).

Chaque année en décembre, lorsqu'il examine le budget de l'année à venir, le Parlement fixe aussi les crédits qui détermineront les dépenses d'APD. L'austérité étant de mise au sein de l'administration fédérale, les dépenses de la coopération au

Tableau $\mathrm{N}^{\circ} 15$

Crédits de programme en vigueur en 1992

\begin{tabular}{|c|c|c|c|}
\hline $\begin{array}{l}\text { Dates } \\
\text { d'approbation }\end{array}$ & Crédits de programme & $\begin{array}{l}\text { Montant } \\
\text { (mio fr.) }\end{array}$ & Durée \\
\hline 04.10 .1990 & $\begin{array}{l}\text { Coopération technique et } \\
\text { aide financière }\end{array}$ & $3 ' 300$ & $\begin{array}{l}4 \text { ans } \\
\text { (dès 19.12.1990) }\end{array}$ \\
\hline 10.12.1991 & Aide humanitaire internationale & $1^{\prime} 050$ & $\begin{array}{l}4 \text { ans } \\
\text { (dès 03.04.1992) }\end{array}$ \\
\hline 30.01 .1991 & $\begin{array}{l}\text { Crédit d'engagement pour } \\
\text { l'aide à la Jordanie, à l'Egypte } \\
\text { et à la Turquie }\end{array}$ & 130 & 4 ans \\
\hline 30.10 .1990 & $\begin{array}{l}\text { Mesures de politique } \\
\text { économique et commerciale }\end{array}$ & 840 & 4 ans \\
\hline 13.03.1991 & $\begin{array}{l}\text { Programmes et projets en faveur } \\
\text { de l'environnement global dans } \\
\text { les pays en développement* }\end{array}$ & 300 & (dès 01.07.1991) \\
\hline 13.03.1991 & $\begin{array}{l}\text { Financement de mesures } \\
\text { de désendettement }\end{array}$ & 400 & (dès 01.07.1991) \\
\hline 29.09 .1987 & $\begin{array}{l}\text { Participation au capital des } \\
\text { banques régionales de } \\
\text { développement }\end{array}$ & 680 & (dès 01.10.1987) \\
\hline 04.10 .1991 & $\begin{array}{l}\text { Adhésion de la Suisse aux } \\
\text { Institutions de Bretton Woods }\end{array}$ & 4'986 & (dès 17.05.1992) \\
\hline
\end{tabular}


développement ont été réduites: le budget de l'APD a subi des réductions linéaires et la durée des crédits-cadres a été prolongée. Les versements de la Suisse aux frais de fonctionnement du Comité international de la Croix-Rouge (CICR), qui a son siège à Genève, constituent toutefois une exception.

La Confédération accorde son aide à quelque 80 pays du Tiers Monde, dont 20 sont des pays de concentration de la coopération suisse au développement. Pour ces derniers, la Suisse a mis en place des programmes à moyen terme et y a installé des bureaux de coordination. En Afrique, ces pays sont les suivants: Mozambique, Tanzanie, Madagascar, Mali, Kenya, Rwanda, Bénin, Burkina Faso, Niger, Tchad; en Amérique centrale et du Sud: Pérou, Bolivie, Équateur, Nicaragua, Honduras; en Asie: Indonésie, Népal, Inde, Bangladesh et Pakistan. La coopération au développement repose sur la loi de 1976 qui oblige la Confédération à soutenir en priorité les pays en développement, les régions et les groupes de population les plus démunis.

La politique suisse en matière de développement relève de la Direction pour la coopération au développement et de l'aide humanitaire (DFAE) et de l'Office fédéral des affaireséconomiques extérieures (DFEP). Une commission mixte-laCommission consultative pour la coopération au développement et l'aide humanitaire - seconde le Conseil fédéral pour ce qui est de la politique Nord-Sud.

\section{Soutien de la démocratisation / bonne gestion gouvernementale}

Ces dernières années, l'attribution d'une aide au développement se décide de plus en plus sur des critères dits de "bonne gestion gouvernementale" (Good Governance). Voici les cinq éléments définis par la Banque mondiale qui sont le gage d'une bonne gestion: transparence (notamment des finances), responsabilité du gouvernement et des autorités locales, pluralisme institutionnel, participation de la population, primauté du droit. A ces cinq éléments, la Suisse ajoute encore les critères suivants: le respect des droits de l'homme, l'intégration des forces de l'ordre dans la société civile, une société ouverte qui favorise l'initiative privée et la décentralisation, la justice sociale qui postule une répartition équitable des richesses et, enfin, une administration compétente, sans corruption (2).

En Suisse, les responsables de l'APD pensent également que la bonne gestion gouvernementale joue un rôle essentiel pour l'aboutissement du processus de démocratisation. Depuis 1986, la DDA mène par exemple en Afrique du Sud le "Programme de mesures positives en vue de l'élimination de l'apartheid" qui soutient des organisations non gouvernementales oeuvrant pour une Afrique du Sud démocratique. Au cours de la période considérée, la DDA a déboursé un demi-million de francs supplémentaires pour financer la préparation des élections. En tant que nouvel instrument de l'APD, l'appui à la tenue d'élections a aussi été appliqué dans d'autres pays. Au Salvador, la DDA et neuf oeuvres suisses d'entraide collaborent à un projet commun ("Initiative sociale pour la démocratie") qui a pour but de motiver la population des régions ravagées par la guerre civile à participer aux élections (prévues pour le printemps 1994). Le principe de bonne gestion compte pour beaucoup dans le choix de la Suisse lors de la mise en oeuvre de mesures de coopération au développement, surtout lorsqu'il s'agit d'actions de désendettement 
ou d'aide à la balance des paiements. La Suisse n'en accorde pas moins une aide à la population des pays où la bonne gestion gouvernementale n'est pas encore de mise, pour ne pas faire subir aux habitants une discrimination encore plus grave en raison des erreurs commises par le gouvernement.

La Conférence annuelle de la DDA de 1993 a également été consacrée aux aspects politiques de la coopération au développement. Elle a eu pour thème: "Politique du développement des années 90: dialogue ou ingérence?" Un dialogue sincère entre partenaires devrait montrer la voie, mais ce dialogue n'est pas aisé et il convient de trouver un nouveau "partenariat des valeurs" (Blankart). Les valeurs occidentales (y compris la bonne gestion gouvernementale) ne doivent pas primer les valeurs traditionnelles ethniques et religieuses d'autres cultures. Le nouveau directeur de la DDA, Walter Fust, a pour la première fois présidé la conférence annuelle.

\section{... et l'économie de marché}

A la base, le Viêt-nam est un pays socialiste, mais il ouvre son économie aux mécanismes du marché libre. Au Viêt-nam, la DDA appuie un programme de formation destiné à des professeurs d'université (dont un tiers sont des femmes), qui a pour but de les familiariser avec les mécanismes de l'économie de marché et de leur enseigner plus particulièrement le rôle et la fonction de l'entreprise. La DDA finance simultanément des séminaires àl'intention des gestionnaires des entreprises publiques - souvent déficitaires. Par ailleurs, elle envisage à l'avenir de coordonner la formation au niveau supérieur dans le domaine de la gestion d'entreprises dans ce pays et de soutenir ainsi les "acteurs du changement".

\section{Soutien des mesures d'ajustement structurel}

Pour soutenir les réformes économiques dans les pays en développement, la Confédération fournit notamment une aide financière immédiate sous forme d'aide à la balance des paiements. Celle-ci est attribuée en tant qu'aide bilatérale ou dans le cadre de cofinancements avec l'AID. Dans ce domaine aussi, la Suisse préfère destiner son aide aux pays les plus démunis et plus particulièrement aux couches de la population les plus touchées par l'ajustement structurel, encourageant ainsi un "ajustement structurel social" (p. ex. en Bolivie, à Madagascar et au Mozambique). En 1992, les dépenses consacrées aux mesures d'ajustement structurel se sont montées à quelque 57 millions de francs. La Suisse encourage avant tout les réformes du secteur financier des pays. La Suisse contribue égalementau "Programme spécial pour l'Afrique" coordonné par I'AID, qui joue un rôle clé dans la coordination de l'aide aux mesures d'ajustement structurel dans les pays africains.

\section{Examen de la coopération suisse au développement par le CAD}

Le Comité d'aide au développement (CAD) de l'OCDE examine tous les deux ans les efforts en faveur du développement fournis par ses 21 membres. En septembre 
1993, la Belgique et la Nouvelle-Zélande ont ainsi analysé l'aide suisse au développement. Comme lors des examens précédents, la qualité de cette aide a été jugée excellente, tandisque sonenvergure demeure modeste. Lahausse substantielle des dépenses d'APD en 1992 doit être mise sur le compte des contributions exceptionnelles versées en 1992 au groupe de la Banque mondiale. Le rapport du CAD rappelle à la Suisse sa promesse d'augmenter les dépenses d'APD pour les porter à $0,40 \%$ du PIB. La Suisse est priée de mettre sa promesse en pratique d'ici à l'an 2000. Pour y parvenir, la Confédération devrait tout mettre en oeuvre pour que le volume de l'aide bilatérale ne soit pas réduit dans les années à venir comme le laissent présager les restrictions budgétaires. Le CAD approuve l'orientation de l'aide vers les pays les plus démunis et vers les couches de populations les plus défavorisées. Il a également loué l'aide bilatérale de la Suisse car elle se caractérise par la participation de la population. L'analyse suggère toutefois une meilleure intégration des femmes ainsi qu'un plus grand respect de l'environnement. La concentration de l'aide - moins de pays, moins de projets - a déjà été proposée à l'occasion des examens préalables et à nouveau évoquée en 1993. Pour que l'aide au développement puisse faire face à des exigences croissantes sans perdre de sa qualité, le CAD suggère dans son rapport une augmentation du personnel de la DDA ainsique des bureaux de coordination. Dans le même temps, il conviendrait de mieux coordonner la politique de développement et les autres domaines de la politique étrangère suisse, afin d'accroître l'efficacité de l'aide au développement. Pour terminer, le CAD apprécie en particulier la création de la facilité suisse de désendettement qui constitue un pas novateur vers la solution de la crise de l'endettement.

Selon la statistique de l'OCDE, 30\% environ des dépenses bilatérales financent des prestations fournies par la Suisse. Celles-ci comprennent avant tout les salaires d'experts et de consultants, mais aussi des mandats découlant de crédits mixtes. L'aide multilatérale profite beaucoup à l'économie suisse, puisque $90 \%$ environ de cette aide sert à financer des commandes adressées à la Suisse; si l'on tient compte des commandes de la Banque mondiale, le total atteint même $120 \%$.

Un rapport de Eurostep-Network (3), dont fait aussi partie la Communauté de travail de cinq oeuvres d'entraide suisses, loue également la qualité et la transparence institutionnelle de l'aide suisse au développement, notamment de celle de la DDA. II regrette par contre l'application de l'aide liée dans le cadre des crédits mixtes ainsi que le retard pris dans l'encouragement des femmes qui est considéré comme un élément essentiel de la lutte contre la pauvreté. II critique également la tendance à accorder plus de moyens à l'aide multilatérale et la pratique selon laquelle le cofinancement de programmes internationaux est encore comptabilisé dans l'aide bilatérale, ce qui donne une allure faussement positive à ce bilan.

\section{Une politique extérieure plus cohérente}

L'expérience des trois dernières décennies de coopération au développement a notamment montré qu'une politique du développement isolée ne peut pas induire des progrès notables dans les pays en développement. C'est pourquoi l'on prône à 
présent un engagement global, dans lequel la coopération au développement n'est qu'un des éléments d'une politique extérieure bien coordonnée. D'un autre côté, l'aide attribuée à un pays doit être mieux coordonnée entre les différents acteurs, ainsi qu'avec les partenaires locaux. La Confédération est consciente de cette faiblesse de la coopération au développement telle qu'elle est pratiquée aujourd'hui et le Conseil fédéral analysera ces problèmes de plus près dans un "rapport Nord-Sud".

\section{Coopération au développement et respect de l'environnement}

Après la Conférence de Rio, la Suisse a réexaminé tous ses programmes de coopération au développement en cours de réalisation pour déterminer leur compatibilité avec les impératifs de l'environnement. Des aménagements ont été et devront être réalisés. Le Conseil fédéral a mis en place un comité interdépartemental chargé de coordonner et de concentrer les politiques sectorielles ayant trait à l'environnement. Le comité élabore un plan d'action national pour la mise en oeuvre del'Agenda21. Les oeuvres d'entraide privées et l'économie privée sont représentées dans divers groupes de travail consacrés à des thèmes précis. (Pour plus de précisions sur l'"après Rio", lire le chapitre 3 ).

A la fin de juillet 1993 , le crédit-cadre destiné à soutenir des programmes en faveur de l'environnement avait déjà été utilisé pour financer 35 projets bilatéraux pour un montant total de 60 millions de francs environ. C'est la DDA qui est responsable de lapartie bilatérale des mesures en faveur de l'environnementet pour le cofinancement en matière d'environnement. 11 projets sont menés dans le domaine climat/énergie, 11 dans le domaine biodiversité/forêt tropicale. Le rapport annuel de 1992 publié conjointement par la DDA et l'OFAEE présente quelques-uns de ces projets: centrale thermique à Sumatra, fabrication d'un frigo écologique en Inde, contrôle des gaz d'échappement en Amérique centrale. L'Office fédéral de l'environnement, des forêts et du paysage (OFEFP) est compétent pour les actions multilatérales en matière d'environnement. Jusqu'à la fin de juillet 1993, la Suisse a engagé 78 millions de francs à titre de contribution au Fonds mondial pour l'environnement (FME, gérée par la Banque mondiale, le PNUD et le PNUE) et au Fonds pour le Protocole de Montréal relatif à des substances qui appauvrissent la couche d'ozone.

\section{Immigration et aide au développement}

La "nomadisation de la pauvreté", la migration économique des habitants de pays pauvres vers les pays riches et le rôle de la coopération au développement préoccupent tous les gouvernements des pays industrialisés. La Suisse a pris part à une réunion organisée sur ce thème par l'OCDE qui s'est tenue à Madrid en avril 1993. Nombre des projets de la coopération suisse au développement ont pour but d'améliorer les conditions de vie des habitants du Tiers Monde, dans l'espoir qu'ils seront ainsi moins tentés à quitter leur pays. Au cours de la réunion on a expliqué que les succès remportés en matière de développement dans les pays du Tiers Monde 
conduisent paradoxalement à un accroissement de l'émigration, en raison d'une meilleure information et d'une plus grande mobilité. La récession a quelque peu freiné la migration économique. Les experts de la réunion de Madrid ont appelé les gouvernements à augmenter massivement leur aide au développement, car même si cette mesure conduit à accroître - temporairement - les migrations, seule l'assurance d'une existence menée dans la dignité et la sécurité offre une garantie optimale contre la "nomadisation de la pauvreté" sous la forme de flux migratoires vers les pays riches.

\section{Aide à la reconstruction au Proche-Orient}

Le Conseil fédéral a décidé de participer à l'aide internationale à la reconstruction au Proche-Orient. II a donc chargé le Département fédéral des affaires étrangères (DFAE) d'ouvrir un crédit-cadre pour financer l'aide à la reconstruction dans la bande de Gaza et en Cisjordanie, d'un montant de 60 millions de francs répartis sur 5 ans. Une première tranche de 15 millions de francs devrait être mise à disposition dès 1994 .

\subsection{Dépenses de l'aide publique au développement en 1992}

En 1992, l'aide de la Suisse aux pays en développement s'est montée à 1'621 millions de francs. La Confédération a versé 1'357 millions de francs et les cantons et les communes 28 millions de francs (ce qui correspond à un total de 1'385 millions de francs à titre d'aide publique au développement, soit $0,39 \%$ du PNB). Les prestations des oeuvres privées d'entraide ont atteint en 1992 quelque 236 millions de francs. Les dépenses d'APD ont nettement augmenté par rapport à l'année précédente (de $27 \%$ ), une augmentation qu'il faut principalement mettre sur le compte de l'adhésion de la Suisse à la Banque mondiale. Cette adhésion a également réduit la part de l'aide bilatérale (79\% en 1991, 69\% en 1992) en faveur de l'aide multilatérale (21\% en $1991,31 \%$ en 1992$)$.

Selon la DDA, la part du PNB consacrée à l'APD atteint 0,39\%. Pour l'OCDE, qui applique d'autres méthodes de comptabilité dans ses statistiques, cette part est de $0,46 \%$. La Suisse ne comptabilise en effet que les versements déjà effectués. Ainsi, tandis que l'OCDE inclut tout le capital de participation au groupe de la Banque mondiale dans la statistique de 1992, la Suisse répartit les versements effectifs sur cinq ans, d'où cette différence d'appréciation. L'aide publique de la Suisse n'est pas remboursable et n'est pas liée, à l'exception des crédits mixtes et de l'aide alimentaire sous forme de produits suisse (lait en poudre). En matière d'aide au développement, I'OCDE distingue l'aide liée, non liée et partiellement liée.

La Direction de la coopération au développement et de l'aide humanitaire (DDA) gère la majeure partie des dépenses d'APD (1'079 millions de francs, 77\% environ). La coopération technique, l'aide financière, l'aide alimentaire et l'aide humanitaire relèvent de sa compétence. En 1992, l'aide financière s'est nettement accrue par rapport à l'année précédente en raison du coût payé par la Suisse pour adhérer au groupe de la Banque mondiale. Ce coût s'élève à 190 millions de francs 
(57 millions à la BIRD, 93 à l'AID et 40 à la SFI). A l'aide fournie par la DDA, viennent s'ajouter 35 millions de francs versés à titre d'aide financière par le Département fédéral des finances aux pays qui ont subi des désavantages économiques en raison de l'embargo décrété pendant la guerre du Golfe (crédit d'engagement spécifique). L'Office fédéral des affaires économiques extérieures (OFAEE) est compétent pour les mesures économiques et commerciales, qui ont coûté 208 millions de francs à la Suisse en 1992. Le tableau 16 indique la répartition des dépenses d'APD de la Confédération.

Tableau $\mathrm{N}^{\circ} 16$

Dépense de l'aide publique au développement (APD)

\begin{tabular}{|c|c|c|c|c|c|c|}
\hline & \multicolumn{4}{|c|}{1992} & \multicolumn{2}{|c|}{1991} \\
\hline & $\begin{array}{c}\text { bilatérale } \\
\text { mio. fr. }\end{array}$ & $\begin{array}{l}\text { multi- } \\
\text { latérale } \\
\text { mio. fr. }\end{array}$ & $\begin{array}{l}\text { Total } \\
\text { mio. fr. }\end{array}$ & $\%$ & $\begin{array}{l}\text { Total } \\
\text { mio. fr. }\end{array}$ & $\%$ \\
\hline Coopération technique & 405 & 96 & 501 & 36 & 479 & 41 \\
\hline Aide financière & 114 & 272 & 386 & 28 & 174 & 15 \\
\hline Mesures économiques & & & & & & \\
\hline et commerciales & 208 & - & 208 & 15 & 231 & 20 \\
\hline Aide alimentaire & 55 & 26 & 81 & 6 & 59 & 5 \\
\hline Aide humanitaire & 151 & 19 & 170 & 12 & 176 & 15 \\
\hline Non classé & 28 & 17 & 45 & 3 & 41 & 4 \\
\hline Remboursement de prêts & & & & & -4 & - \\
\hline Total & 955 & 430 & 1'386 & 100 & 1156 & 100 \\
\hline En pourcentage du PNB & & & 0,39 & & 0,34 & \\
\hline Source: & de l'o & EE 199 & & & & \\
\hline
\end{tabular}

\subsection{Coopération au développement bilatérale}

L'aide bilatérale représente la majeure partie de l'aide suisse au développement. En 1992, cette forme d'aide s'est montée à 955 millions de francs, soit $70 \%$ environ de l'aide totale. Pour des raisons déjà mentionnées (adhésion de la Suisse à la Banque mondiale), la part de cette aide a diminué par rapport à l'année précédente $(79 \%)$. A l'avenir aussi, en raison de l'engagement financier constant de la Suisse en tant que membre du groupe de la Banque mondiale, on devra s'attendre à voir la part de l'aide multilatérale s'accroître aux dépens de l'aide bilatérale dont la part restera constante ou ira s'amenuisant. L'inscription des cofinancements avecl'AID (1991:43 millions de francs; 1992: 41 millions de francs) dans l'aide bilatérale fait l'objet de critiques depuis des années. Pour justifier sa façon de faire, la Confédération argue 
que les cofinancements interviennent certes dans un contexte multilatéral, mais que la Suisse participe toutefois au choix des projets et des pays bénéficiaires.

Les oeuvres privées d'entraide et l'économie privée sont des partenaires de premier plan de la Confédération dans la réalisation de projets et de programmes bilatéraux. Elles se chargent en effet de gérer en régie des actions de la coopération au développement ou reçoivent des contributions fédérales pour leurs propres projets. En 1992, la Confédération a versé 174 millions de francs aux oeuvres d'entraide privées pour leur propres actions ou pour des projets en régie; les versements des cantons et des communes ont atteint 26 millions de francs.

\section{Répartition régionale}

Depuis des années, la répartition régionale de la coopération bilatérale demeure pour ainsi dire inchangée. Les pays en développement africains s'arrogent la plus grande part avec 355 millions de francs (37\%), l'Asie reçoit 253 millions de francs (26\%), l'Amérique latine 117 millions de francs (12\%) et l'Europe (y compris l'Albanie et l'ex-Yougoslavie) 37 millions de francs (4\%). La répartition géographique de l'aide correspond à limportance des continents dans la liste des principaux pays bénéficiaires.

Dans la répartition par revenu, les pays en développement les moins avancés (revenu inférieur à 765 dollars) reçoivent $60 \%$ de l'aide suisse au développement et $20 \%$ vont aux pays à revenu moyen (les $20 \%$ restants ne sont pas répartis).

\section{Répartition sectorielle}

La coopération au développement bilatérale de la DDA s'effectue dans les secteurs suivants: agriculture, élevage (17\%); infrastructure, eau, énergie (18\%); forêt, environnement ( $9 \%)$; santé, nutrition, population ( $9 \%)$; éducation, formation, culture $(8 \%)$; artisanat, industrie, commerce $(6 \%)$; économie globale, finances, services (5\%); politique sociale, administration, justice (2\%). Un quart environ de l'aide bilatérale gérée par la DDA est multisectorielle.

A l'avenir, la Confédération désire réduire les programmes géographiques et sectoriels. Cette intention est motivée par le besoin de réduire les dépenses mais aussi par des considérations relevant de l'efficacité. La DDA examine de son côté une meilleure mise en valeur des connaissances et des capacités à disposition, dans le butd'améliorer encore la collaboration, ou la répartition du travail, entre Confédération et oeuvres d'entraide privées. Cette évolution n'ira pas sans la restructuration de certains secteurs de l'administration. Dans un deuxième temps, on soumettra à une évaluation les structures des programmes nationaux dans les principaux pays bénéficiaires, ainsi que les bureaux de coordination.

\subsection{Coopération au développement multilatérale}

Les organisations des Nations Unies, les banques de développement régionales et le groupe de la Banque mondiale ainsi que des organisations internationales 
n'appartenantpas àla famille onusienne(CICR, Fonds international de développement agricole (FIDA), entre autres) sont les principaux partenaires de la coopération multilatérale de la Confédération. Suite à l'adhésion de la Suisse à la Banque mondiale et en raison des obligations financières que cela implique, la part de l'aide multilatérale de la Suisse est appelée à s'accroître (244 millions de francs en 1991, $21 \%$ de l'aide; 430 millions en 1992, soit $31 \%$ ). Les organismes de développement privés ne voient pas cette tendance d'un bon oeil. Les oeuvres d'entraide accordent beaucoup d'importance à l'aide bilatérale, car cette forme d'aide permet à la Suisse de suivre, de contrôler et d'évaluer un projet, ce qui n'est pas possible dans le cadre de l'aide multilatérale. Puisque la Confédération est confrontée à des problèmes budgétaires, les oeuvres d'entraide craignentquel'accroissement de l'aide multilatérale n'intervienne aux dépens de programmes bilatéraux et rappellent au Conseil fédéral les promesses faites avant les votations sur l'adhésion aux institutions de Bretton Woods.

Parmi les critères qui régissent la participation de l'aide suisse à des actions multilatérales on trouve notamment l'importance de l'organisation concernée pour la coopération au développement internationale ainsi que l'efficacité de l'aide fournie. Dans le cadre de l'aide multilatérale, la Suisse insiste également pour que l'aide s'adresse aux pays les plus pauvres ainsi qu'aux groupes de la population les plus défavorisés. Elle préfère donc collaborer avec des organisations qui s'attachent à lutter contre la pauvreté. De plus, la Suisse souhaite prendre part activement aux décisions etàl'évaluation des organisations et, lorsquec'est possible, être représentée dans les organes décisionnels.

Le principal partenaire de la Suisse parmi les institutions de l'ONU est le Programme des Nations Unies pour le développement (PNUD) qui reçoit 60 millions de francs, soit la contribution la plus élevée. La Suisse accorde par ailleurs d'importantes contributions au Programme alimentaire mondial (PAM) et au Fonds des Nations Unies pour l'enfance (UNICEF), au Fonds des Nations Unies pour les activités en matière de population (FNUAP) et au Fonds d'équipement des Nation Unies (FENU).

En 1992, le groupe de la Banque mondiale, les banques régionales de développement et leurs fonds spéciaux ont reçu au total plus de 250 millions de francs, contre seulement 71 millions l'année précédente. Comme nous l'avons déjà maintes fois mentionné, ce montant s'explique par le coût de l'adhésion de la Suisse à la Banque mondiale. La Confédération soutient aussi la recherche agricole internationale en cofinançant le Groupe consultatif (GCRAI) par des contributions annuelles de 10 millions de francs environ. Le GCRAl est l'organe suprême qui dirige 18 centre internationaux de recherche agricole. Il est financé par 41 pays donateurs et parrainé par la Banque mondiale, la FAO et le PNUD. La Suisse est membre actif du Groupe consultatif depuis 1972. (Toutes les contributions de la Suisse aux organisations internationales sont détaillées dans la 3ème partie du présent annuaire consacrée aux statistiques en matière de coopération au développement.) 
Dans le cadre des activités multilatérales, la Suisse a pris part à d'importantes conférences au cours de la période considérée: Conférence de Rio sur l'environnement et le développement, Conférence internationale sur la nutrition, Conférence sur les droits de l'homme et le développement. Ces diverses conférences font l'objet de chapitres séparés.

AID: Déjà avant d'adhérer au groupe de la Banque mondiale, la Suisse soutenait régulièrement l'Association internationale de développement (AID), non pas en lui versant des contributions globales, comme elle devra le faire à l'avenir auprès du groupe de la Banque mondiale, mais en participant à des cofinancements. Dans la statistique suisse, ces participations figurent dans l'aide bilatérale, alors que le soutient à l'AID (participation à l'AID-10) devrait à l'avenir, selon la classification internationale, figurer parmi les dépenses multilatérales.

\subsection{Coopération technique et aide financière}

La coopération technique comprend tous les éléments d'un projet bilatéral, c'està-dire le soutien technique (experts, boursiers), mais aussi le capital et le matériel nécessaires à la réalisation du projet. Elle comprend également les montants versés à des organisations privées en Suisse et à des organisations locales dans les pays en développement. La coopération technique de la Confédération englobe donc aussi des éléments de l'aide financière. Depuis peu, la coopération technique de la Confédération tend à s'orienter vers un accroissement du soutien direct de la population et vers une plus grande participation à des fonds sociaux destinés à compenser la dégradation des conditions de vie engendrée par les mesures d'ajustement structurel. A l'avenir, la Confédération souhaite motiver la population concernée à participer à la réalisation et à l'évaluation des projets. Concrètement, le nombre d'experts suisses envoyés sur place a diminué et ils ont été remplacés par des spécialistes locaux. La DDA fait par contre plus souvent appel aux services ponctuels de consultants. L'aide financière se traduit par des investissements dans un projet ou un programme, qui est en règle générale géré directement par le gouvernement du pays partenaire. La différence entre coopération technique et aide financière relève de plus en plus de motifs historiques et de nature réglementaire. En réalité, ces formes de coopération au développement visent toutes deux à responsabiliser le partenaire et à promouvoir des projats globaux de développement.

En 1992, le Conseil fédéral a destiné 501 millions de francs (36\% des dépenses d'APD) à la coopération technique (contre 478 millions l'année précédente). Ce domaine où l'aide est essentiellement bilatérale est le cadre traditionnel de la majeure partie de la coopération suisse au développement. Les montants destinés à l'aide financière ont atteint en 1992386 millions de francs (28\%), contre 174 millions de francs en 1991. Les chiffres de 1992 incluent notamment les frais d'adhésion au groupe de la Banque mondiale de 191 millions de francs, ainsi que l'aide financière de 35 millions de francs destinée aux pays de la région du Golfe (Jordanie: 26,7 millions de francs, Turquie: 7,2 millions, Égypte: 0,7 million). 


\subsection{Mesures de politique économique et commerciale}

Les mesures de la politique de développement dans les domaines économique et commercial comprennentlapromotion du commerce avec les pays en développement, la politique concernant les produits, de base, dont la compensation des pertes de recettes d'exportation, la promotion de l'industrialisation par le transfertde technologie, la stimulation du secteur privé, l'aide financière sous forme de financements mixtes et aide à la balance des paiements, ainsi que les actions de désendettement. C'est I'Office fédéral des affaires économiques extérieures (OFAEE) qui est compétent pour ce domaine de l'aide. En 1992, les dépenses effectuées dans ce domaine ont reculé par rapport à l'année précédente: elles se sont montées à 208 millions de francs (15\% des dépenses d'APD) en 1992 et à 231 millions de francs (20\%) en 1991 .

\section{Financement mixte}

Les crédits mixtes - un instrument classique de la coopération au développement servent à mobiliser des moyens privés supplémentaires pour financer des projets industriels ou d'infrastructure. Ils contribuent à couvrir les grands besoins financiers des pays en développement qui n'ont qu'un accès limité aux marchés internationaux des capitaux, et ils stimulent dans le même temps les exportations suisses, car l'utilisation des moyens fournis est liée à des livraisons provenant de Suisse. La part fédérale des crédits mixtes constituant un don, ceux-ci offrent aux pays bénéficiaires des conditions plus favorables que les crédits ordinaires. La part fédérale est attribuée à titre de don depuis 1988, tandis que la part bancaire est accordée aux conditions du marché. Outre l'aide alimentaire, les crédits mixtes sont la seule forme d'aide liée que la coopération suisse au développement inscrit comme telle dans ses statistiques. Selon l'OCDE, la part de l'aide liée suisse est plus importante, car l'organisation classe le travail des experts, une partie de la promotion des exportations et d'autres mesures de la coopération au développement parmi l'aide liée ou partiellement liée.

Depuis 1977, la Suisse a conclu au total 36 accords portant sur des financements mixtes en faveur de 21 pays en développement et de deux banques régionales de développement. Le total de ces crédits dépasse 2 milliards de francs. La part publique de 776 millions de francs se situe entre 6 et $8 \%$ de l'aide publique suisse au développement et couvre environ $1 \%$ des exportations suisses vers les pays en développement.

Les nouvelles directives sur l'octroi de crédits d'aide au développement adoptées dans le cadre de l'OCDE ("directives de Helsinki") sont entrées en vigueur en 1992. Elles restreignent sensiblement l'octroi de nouveaux financements mixtes par la Suisse. La Suisse a approuvé cette nouvelle réglementation car elle correspond aux objectifs et aux intérêts de la politique suisse du développement et du commerce extérieur et qu'elle réduit notamment les distorsions commerciales internationales et assure une distribution plus efficace de l'aide. Pour appliquer ces nouvelles règles, il importe donc de réorienter les instruments suisses de financement. Une telle réorientation est d'ailleurs toute indiquée si l'on considère les bouleversements qui 
ont marqué l'économie et la politique des pays en développement et le rôle que joue le secteur privé dans la croissance économique. L'OFAEE élabore actuellement de nouveaux instruments destinés à soutenir le processus de libéralisation et de privatisation dans les pays en développement et à promouvoir les investissements privés.

Un seul accord portant sur un financement mixte a été conclu en 1992: la Société andine de développement (SAD) s'est vu accorder un crédit mixte de 20 millions de francs, dont 7 millions de part fédérale.

Un premier crédit portant sur un financement mixte a été conclu avec le Viêt-nam en 1993. II porte sur un montant de 25 millions de francs (la part fédérale est de $50 \%$ ). Les financements mixtes conclus avec la Thaïlande et le Pakistan ont été augmentés de 14 millions de francs (un tiers de part fédérale) et de 21 millions de francs (40\% de part fédérale) respectivement. En 1993, toujours, a été achevée la conversion des parts fédérales accordées par le passé sous la forme de prêts remboursables. Cette conversion a porté sur 300 millions de francs et concerne 13 pays en développement.

\section{Aide à la balance des paiements}

En 1992, des aides à la balance des paiements ont été accordées à l'Ouganda, au Cameroun, au Salvador, à la Tanzanie et au Mozambique sous forme de cofinancements avec la Banque mondiale (15 millions de francs pour l'Ouganda, 12,5 millions pour le Cameroun), avec la Banque interaméricaine de développement ( 10 millions de francs pour le Salvador) ou sous forme bilatérale ( 9 millions de francs pour la Tanzanie, 2 millions de francs pour le Mozambique). En 1993, des aides à la balance des paiements ont été conclues avec l'Ethiopie ( 10 millions de francs en cofinancement avec l'AID), avec le Viêt-nam (15 millions de francs sur une base bilatérale) et avec Madagascar (1 million de francs à titre d'aide bilatérale).

\section{Mesures de désendettement}

Pour 1992, 59,5 millions de francs sont inscrits dans la statistique de la DDA au titre de mesures de désendettement. Les mesures prises sont décrites plus en détail au chapitre II. 1.7.

\section{Produits de base}

La Suisse compense les pertes de recettes d'exportation que subissent les pays en développement en raison de la baisse des prix des produits de base. En 1992, un total de 18 millions de francs ont ainsi été versés à l'Ethiopie, à la Gambie, au Mali et au Tchad. Ces paiements ont servi à améliorer la structure de production et de commercialisation du café, des arachides et du coton. En 1993,6,5 millions de francs de paiements compensatoires ont été versés à l'Ethiopie, à la Gambie, à l'Ouganda et au Soudan. En Suisse, la Confédération a soutenu l'introduction du label de café "Max Havelaar" dont la vente assure le versement d'un prix plus équitable aux producteurs de café. 


\section{Promotion commerciale}

En 1992, la Confédération a consacré 5,1 millions de francs à la promotion commerciale. LaSuisse a réduit ses contributions au Centre de commerce international de Genève placé sous la responsabilité de la CNUCED et du GATT; elles sont passées de 3,5 millions de francs en 1991 à 2,4 millions de francs en 1993. Le Centre de commerce international vise à promouvoir la gestion des exportations et des importations dans les pays en développement. II traverse actuellement une crise interne et politique qui a incité la Suisse à réduire ses contributions jusqu'à ce que les problèmes soient résolus et que le Centre puisse reprendre une activité efficace.

\section{Industrialisation}

Pour encourager l'industrialisation dans les pays en développement, la Confédération soutient en premier lieu le Bureau de l'ONUDI à Zurich, qui encourage les investissements suisses dans les pays en développement et, depuis 1990, aussi dans les pays d'Europe de l'Est.

\subsection{Aide humanitaire}

L'aide humanitaire est destinée à atténuer la gravité des situations d'urgence grâce à l'aide alimentaire, l'aide aux réfugiés et à l'aide en cas de catastrophe. Les actions de l'aide humanitaire et de l'aide alimentaire relèvent de la compétence de la DDA. En 1992, les dépenses de l'aide humanitaire se sont montées à 170 millions de francs (contre 162 millions l'année précédente), dont la majeure partie (151 millions de francs) à titre bilatéral. L'aide alimentaire a absorbé 81 millions de francs (74 en 1991), dont 55 millions sous forme bilatérale. L'aide humanitaire couvre le monde entier. Comme les années précédentes, la répartition régionale place l'Afrique au premier rang des bénéficiaires (30\%); $14 \%$ de l'aide humanitaire suisse ont été consacrés à l'Europe (ex-Yougoslavie, Albanie).

Le Comité international de la Croix-Rouge (CICR) est le principal partenaire de la Confédération en matière d'aide humanitaire. En 1992, la Suisse a versé des contributions d'un montant de 80 millions de francs, dont 55 millions au budget du siège (sur la base d'un arrêté fédéral spécifique). En 1993, le Parlement a accepté une proposition du Conseil fédéral d'augmenter les contributions suisses au budget du siège du CICR et de les faire passer à 60 millions annuels pour 1994 et 1995 et à 65 millions pour 1996 et 1997 . A l'initiative du CICR, la Suisse a convié tous les pays du monde à Genève pour participer à la Conférence internationale pour la protection des victimes de la guerre. Celle-ci s'est déroulée en août 1993 et a suscité un grand écho (cf. chap. II 4.2).

Les autres grands partenaires de l'aide humanitaire suisse sont:

- Le Programme alimentaire mondial, PAM, (38 millions de francs). Le PAM est un organe spécial de la FAO qui a été créé en 1963 avec la tâche précise de faire parvenir aux pays en développement les excédents alimentaires des pays 
industrialisés. Aujourd'hui, le PAM gère le plus gros budget de toutes les institutions spécialisées des Nations Unies et fournit avant tout une aide d'urgence en cas de famine.

- Le Commissariat des Nations Unies pour les réfugiés, UNHCR, (33 millions de francs).

- L'Office de secours et des travaux des Nations Unies pour les réfugiés de Palestine dans le Proche-Orient, UNRWA, (11 millions de francs). Cet office fournit depuis 40 ans une aide aux réfugiés palestiniens. Un accord de paix ayant été conclu en 1993, l'aide à la reconstruction deviendra prioritaire. En 1993, la Suisse a accordé 3 millions de francs à titre d'aide immédiate à des projets de I'UNRWA pour la réouverture d'écoles et de centres de santé.

- La Croix-Rouge suisse, CRS, a reçu en 1992 un soutien de 9 millions de francs.

\section{Notes}

1. Dans le présent chapitre, nous passons en revue les aspects importants de la coopération au développement en 1992 et 1993 (jusqu'à fin septembre 1993). Les statistiques détaillées sur les flux financiers vers les pays en développement sont présentées et commentées dans la troisième partie de l'Annuaire. Des chapitres particuliers abordent les mesures de désendettement et l'adhésion à la Banque mondiale et au FMI (chap. II 1), le problème de l'environnement (chap. II 3), les conférences internationales (chap. II 4 et II 5), la coopération en matière de recherche (chap. IV) et l'aide aux pays de l'Est (chap. VII). Dans son article publié dans la deuxième partie du présent Annuaire, Rudolf Dannecker, vice-directeur de la DDA, présente les nouvelles tendances que l'évolution du contexte national et international a fait apparaître dans la coopération bilatérale au développement de la Suisse, notamment en raison de la récession et des restrictions budgétaires imposées à la coopération au développement.

2. Le vice-président de la Banque mondiale, Ismail Serageldin, a expliqué les principes de bonne gestion gouvernementale appliqués par la Banque à l'occasion de la Conférence annuelle de la DDA à Berne, le 6 septembre 1993. Dans son discours prononcé à la même conférence, le Conseiller fédéral Flavio Cotti a ajouté, en décrivant le principe de bonne gestion, les autres éléments exigés par la Suisse.

3. L'Eurostep-Network réunit 22 organismes non gouvernementaux de 15 pays dont la Communauté de travail Swissaid, Action de Carême, Pain pour le prochain, Helvetas, Caritas. Dans sont rapport "The Reality of Aid. An independent Review of international Aid" (juin 1993), l'organisation passe en revue l'aide fournie par les différents pays selon la statistique de l'OCDE. Cette étude peut être obtenue auprès de la Communauté des oeuvres d'entraide à Berne.

\section{Sources}

DDAVFAEE, Coopération au développement de la Confédération suisse, Rapport annuel 1992

DDANOFAEE, MEMORANDUM 1992 de la Suisse au Comité d'aide au développement de I'OCDE, Berne, juillet 1993 
OCDE, Examen de l'aide de la Suisse par le CAD, Paris, septembre 1993 IUED (éd.)/DDA, Aide suisse aux pays en développement et aux pays de l'Europe de l'Est 1992, Genève, octobre 1993

Rapport sur la politique économique extérieure 1992

Service de presse de la DDA: SP 20 septembre 1993 (La Suisse appuie les élections en Afrique du Sud / Processus de paix au Salvador / La DDA soutient la formation de managers au Viêt-nam); SP 19 juillet 1993 (Crédit-cadre pour le financement de projets d'environnement)

René Holenstein, Neokonservative Entwicklungsstrategien - Schliesst sich der Kreis?, mosquito, no 7/octobre 1993

Communiqué de presse du DFAE, 20.9.1993 (Aide à la reconstruction au Proche-Orient) NZZ, 29.9.1993 (Le Conseil des Etats débat de la contribution suisse au CICR)

NZZ et Tages-Anzeiger, 7.9.1993 (Conférence annuelle de la DDA)

Tages-Anzeiger, 2.4.1993 (Conférence de l'OCDE à Madrid sur l'immigration et la coopération au développement)

\section{Aide privée au développement}

\subsection{Dépenses en 1992}

En 1992, les organismes suisses privés de coopération au développement ont dépensé 236 millions de francs pour l'aide aux pays en développement (soit 14,6\% du total de l'aide suisse, sans les pays de l'Est). Si l'on tient compte des 174 millions de francs que la Confédération a versés aux oeuvres d'entraide pour financer leurs propres actions et les projets exécutés en régie, ainsi que des 26 millions de francs alloués par les cantons et les communes, les dépenses totales de l'aide privée ont atteint $\mathbf{4 3 6}$ millions de francs, soit $\mathbf{2 7 \%}$ de l'aide suisse au développement.

Par rapport aux 192 millions de francs de l'année précédente, les dépenses privées ont nettement augmenté et ce de quelque $23 \%$. Cette hausse est d'autant plus surprenante que l'on craignait que les Suisses se montreraient moins généreux pour les pays en développement en période de crise économique (1). Elle s'explique d'une part par le sentiment de solidarité avec les personnes vivant dans la pauvreté, mais aussiparl'accroissementdu nombre de catastrophes: déplacementetoppression des Kurdes au Nord de l'Irak et en Turquie, faim en Afrique, réfugiés et victimes de la guerre dans l'ancienne Yougoslavie, pour ne citer que quelques-unes des collectes organisées. Alors que quelques oeuvres d'entraide - surtout les plus importantes - ont enregistré une augmentation des dons, les autres organismes de coopération au développement doivent faire face à un recul des rentrées financières. Sur les 236 millions de francs dépensés pour l'aide privée au développement, 173 millions de francs (73\%) ont été consacrés à la coopération au développement et 63 millions de francs (27\%) à l'aide humanitaire. 
L'aide aux pays de l'Est (sans l'ancienne Yougoslavie qui est classée parmi les pays en développement) a atteint 19 millions de francs (contre 21 millions l'année précédente). De cette somme, 7,4 millions de francs ont été dépensés à titre d'aide humanitaire. La part de l'aide humanitaire aux pays de l'Est a nettement diminué au profit d'une aide accrue au développement.

En Suisse, nombre d'institutions participent à l'aide privée au développement: selon les statistiques, 209 organisations ont fourni en 1992 de l'aide aux pays du Sud. La majeure partie de l'aide provient cependant des organismes les plus importants: en 1992, 9 oeuvres d'entraide ont géré plus de la moitié des dons offerts par la population.

\subsection{Thèmes prioritaires}

Les grandes oeuvres d'entraide, mais aussi les nombreuses organisations plus petites et très petites, mènent leurs propres projets de coopération avec des partenaires du Sud et des pays de l'Est. L'aide privée au développement vise aussi les couches de population les plus pauvres et recherche la participation des personnes concernées. Outre la coopération au développement concrète, les organisations privées accordent une grande importance - celle-ci varie toutefois selon leur conception - à la diffusion d'informations auprès du public suisse. Certaines organisations (Déclaration de Berne, Action place financière) se consacrent exclusivement à la sensibilisation des citoyens suisses.

Les cinq grandes oeuvres d'entraide Swissaid, Action de Carême, Pain pour le prochain, Helvetas et Caritas (membre depuis 1992) sont regroupées au sein d'une Communauté de travail qui a son propre centre de documentation et d'information. La Communauté de travail prend position sur les principaux aspects de la politique du développement et elle est représentée au sein de la Commission consultative pour la politique du développement ainsi que dans divers groupes de travail fédéraux. Au cours de la période considérée, la Communauté a surtout analysé l'adhésion de la Suisse au FMI et à la Banque mondiale, ainsi que l'attitude des représentants suisses auprès des institutions de Bretton Woods. Les autres thèmes prioritaires comprenaient le volume et la qualité de l'aide publique au développement, les mesures de désendettement, la mise en oeuvre des promesses faites lors de la Conférence sur l'environnement, ainsi que les négociations multilatérales du GATT et la coopération avec les pays de l'Est (voir les chapitres consacrés à ces sujets).

Nous détaillons ci-après les thèmes prioritaires des activités de quelques organisations:

Dix oeuvres suisses d'entraide, soutenues par la DDA, ont mené en 1993 une campagne pour les Adivasi dans le cadre de l'"Année internationale des peuples autochtones". Les Adivasi sont les premiers habitants de l'Inde. Les 60 millions d'Adivasi sont répartis entre 255 peuples. Aujourd'hui, ils appartiennent aux couches de population qui souffrent le plus des violations des droits de l'homme en Inde. Ils se trouvent tout en bas de l'échelle des castes indienne. 
En 1993, la collecte de fonds de Swissaid a eu pour thème "Le maïs, c'est la vie". Elle souhaitait attirer l'attention sur la nécessité de sauvegarder la diversité des espèces et sur la diversification de l'agriculture. Chaque automne, Swissaid organise à l'intention de ses collaborateurs et des personnes intéressées un symposium consacré à un thème spécifique de la coopération au développement. La réunion organisée en octobre 1993 a traité du travail et des perspectives de la recherche agricole publique et internationale. II existe en tout 18 centres internationaux de recherche qui se consacrent à l'étude de divers aliments de base (riz, blé, pommes de terre), mais abordent aussides problèmestechnologiques (technologie agricole, biotechnologie). Leur travail allant croissant et leurs moyens étant limités, ces centres devront mieux coordonner et concentrer leur travail s'ils souhaitent assumer à long terme les tâches qui leur incombent. Leurs travaux jouent en effet un rôle primordial dans la coopération au développement (sécurité de la nutrition, luttecontre lapauvreté et protection desespèces). La Suisse soutient la recherche agricole internationale notamment en allouant des contributions au Groupe consultatif GCRAI.

La campagne de collecte d'Helvetas s'est déroulée en 1993 sous une devise en faveur de la protection des autochtones de la planète, ceci dans le cadre de l'"Année internationale des peuples autochtones". En 1992, Helvetas s'est donné une nouvelle image directrice qui comprend les principes de base à appliquer en matière de développement: le principe fondamental, le principe d'auto-assistance, le principe d'équité, le principe d'autonomie et le principe de partenariat. Helvetas se chargera en outre de réaliser les projets de construction de logements qui seront financés par la collecte de fonds spéciale organisée en 1993 par l'Union suisse pour l'amélioration du logement (organisation faîtière qui regroupe 750 coopératives de logement) à l'occasion de son 75ème anniversaire. L'Union a l'intention de consacrer 0,5 million de francs à la construction de logements dans le Tiers Monde. Voilà un exemple parmi tant d'autres de petits projets d'aide au développement issus de l'initiative privée. Un producteur de fromages (Baer) a prélevé en 1993 un supplément sur le prix d'un de ses produits qui servira à alimenter un fonds spécial destiné à financer des projets de développement. Le premier projet ainsi financé est la construction de cuisines communautaires au Mexique; le travail sera coordonné par l'UNICEF.

Caritas Suisse, la plus grande oeuvre d'entraide de Suisse, souhaite à l'avenir faire preuve d'un plus grand engagement social pour soutenir plus activement la politique de réforme-également en Suisse. Cette oeuvre d'entraide confessionnelle fournit un travail social en Suisse (aide aux nouveaux pauvres, programmes pour les chômeurs, prise en charge de réfugiés) et gère des projets dans des pays en développement. Au-delà de son travail d'utilité publique, Caritas veut faire face aux exigences d'une politique de réforme efficace qui ne doit pas "se limiter à quelques adaptations inévitables du système". Elle veut "s'engager dans les contradictions du modernisme ambiant" et prendre part à la réorganisation du monde "pour contribuer à la naissance d'une nouvelle solidarité" (2). Les collectes de fonds menées en 1993 par Pain pour le prochain et Action de Carême, également des organisations confessionnelles, ont eu pour thème "Vive l'Afrique!" Ces deux oeuvres d'entraide soutiennent la démocratisation dans les pays africains et leurs efforts pour améliorer la situation matérielle de la population. 
En 1993, la Journée mondiale de l'alimentation (16 octobre) s'est déroulée en Suisse sous la devise "La biodiversité, c'est la vie". C'est l'organisation de développement Déclaration de Berne (DB) qui coordonne chaque année les actions menées à l'occasion de cette journée. Elle a deux sections en Suisse, une pour la Suisse alémanique (Zurich) et une pour la Suisse romande (Lausanne). La DB a été fondée en 1968 avec le but de sensibiliser la population suisse aux relations entre les pays riches du Nord et les pays exploités du Sud. En 1993, à l'occasion de son 25ème anniversaire, la DB a fait le bilan de ses activités. Comme toutes les organisations oeuvrant pour la coopération entre le Nord et le Sud, la DB constate qu'après trois décennies d'aide au développement la différence de niveau de vie entre le Nord et le Sud s'est encore accrue et que la lutte contre la pauvreté prend aujourd'hui et prendra demain une dimension encore plus vaste. Conclusion du bilan après 25 ans d'existence: "On a encore besoin de nous!" (Vers un développement solidaire, juillet 1993/no 118).

Depuis quatre ans, Swisscontact, une fondation privée pour la coopération technique au développement, voit diminuer les donsqui luisont adressés. Swisscontact travaille avec divers pays en développement dans le domaine de l'adaptation technologique et, plus récemment aussi, dans celui de la technologie de l'environnement. A l'avenir, elle souhaite lancer des projets dans les pays en développement de l'Est, notamment dans les quatre pays de la CEI (Azerbaïdjan, Turkménistan, Ouzbékistan et Kirghizistan) que la Suisse représente au sein du FMI. En Amérique latine, la fondation gère un programme international (Costa Rica, Guatemala, El Salvador) visant à réduire les gaz d'échappement des moteurs à combustion. Dans le cadre de ce programme, les propriétaires de garages suivent une formation pour pouvoir transmettre certaines connaissances à leurs apprentis. La fondation, financée avant tout par des dons de l'industrie privée, voit depuis quatre ans diminuer les dons qui lui sont adressés, ce recul est en partie compensé par une augmentation des contributions de la Confédération.

La pauvreté, l'exode rural, le manque de formation, l'absence de perspectives d'emploi et la pression croissante de la consommation conduisent nombre de femmes et d'enfants des pays en développement (en Thaïlande, au Sri Lanka) à se prostituer, notamment avec les touristes des pays industrialisés. En 1993, les deux organisations de coopération au développement que sont Terre des hommes Suisse et Groupe de travail tourisme et développement ont mené conjointement en Suisse une campagne contre la prostitution des enfants et publié une documentation à ce sujet (3). Elles demandent que la Suisse et les autres pays européens concluent des accords bilatéraux avec les pays visés par le tourisme sexuel qui permettraient de punir en Suisse les délits commis sur place. La Thaïlande est une destination de vacances très prisée: quelque 75000 Suisses se rendent en effet chaque année dans ce pays. Terre des hommes a consacré sa collecte de fonds de 1993 aux enfants victimes de la guerre.

Le Fonds de solidarité pour la libération sociale dans le tiers monde, Solifonds, a fêté en 1993 ses dix années d'existence. La fondation a été créée en 1983 par le Partisocialiste suisse, I'Union syndicale suisse etl'Oeuvre suisse d'entraide ouvrière. Elle a pour tâche de soutenir le combat politique (notamment celui des syndicats) 
pour la justice et la libération sociale dans les pays en développement, tout en sachant que la justice sociale est une condition préalable à tout processus de développement. Depuis qu'il existe, le Solifonds a appuyé nombre de grèves, de campagnes politiques et d'actions en faveur du respect des droits de l'homme.

La Croix-Rouge suisse, CRS, oeuvre d'une part pour la formation en Suisse (formation professionnelle dans le domaine de la santé, travail social, prise en charge de réfugiés) et fournit, d'autre part, une aide d'urgence et de reconstruction dans les zones de conflit et dans les pays en développement, en concentrant ses efforts sur l'approvisionnement de base dans le domaine médical. La Croix-Rouge reçoit d'importantes subventions de la Confédération (57 millions de francs pour 1992), mais administre également de l'argent provenant de collectes de fonds de la Chaîne du bonheur dans le cadre de certaines actions en cas de catastrophe. La collecte annuelle menée en mai 1993 avait pour thème: "La bonne volonté donne des ailes", pour montrer que l'"aide est un acte spirituel".

\section{Notes}

1. L'augmentation de l'aide privée au développement s'explique d'une part par l'augmentation des fonds recueillis lors des collectes, bien que les résultats de ces collectes varient sensiblement d'une oeuvre d'entraide à l'autre. D'autre part, l'enquête sur l'aide privée suisse au développement a réuni des données sur un plus grand nombre d'organisations que l'année précédente (209 pour 1992 contre 193 pour 1991). Les prestations des organisations qui avaient aussi répondu l'année précédente se sont accrues de $14 \%$. Les chiffres sont tirés du rapport Aide suisse aux pays en développement et aux pays de l'Europe de l'Est (Genève, octobre 1993), publié par l'IUED sur mandat de la DDA.

2. Propos tenus par le directeur de Caritas, Jürg Krummenacher, à l'occasion du Forum "La Suisse demain" du 21 avril 1993, cités dans le Tages-Anzeiger du 22.4.1993.

3. La documentation Gebunden im Schweigen - Sex mit Kindern ist ein Verbrechen résume les principaux résultats de la campagne lancée il y a deux ans. Elle peut être obtenue auprès de Terre des hommes Suisse et du Groupe de travail tourisme et développement, à Bâle.

\section{Sources}

IUED/DDA (éd.), Aide suisse aux pays en développement et aux pays de l'Europe de l'Est 1992, Genève, octobre 1993

Helvetas, Ureinwohner, numéro spécial de "Partnerschaft", septembre 1993

Déclaration de Berne, Vers un développement solidaire, numéros de 1992 et 1993

Service de presse de la DDA, SP 19/juillet 1993 (La campagne pour les Adivasi)

Image directrice d'Helvetas, avril 1992

Tages-Anzeiger, 19.5.1993 (Caritas: plus de dons malgré la récession)

OSEO et Solifonds, Solidarité sans frontières - Joumal du premier mai (Dix ans de Solifonds)

NZZ, 20.10.1993 (Le symposium d'automne de Swissaid)

NZZ, 3.5.1993 (Fonds recueillis par Swisscontact en baisse)

NZZ, 6.9.1993 (Solidarité dans la construction de logements)

NZZ, 7.5.1993 (Collecte de fonds annuelle de la Croix-Rouge suisse) 